\title{
Oversampled Channelized Receiver for Transmitted Reference UWB System in the Presence of Narrowband Interference
}

\author{
Lei Feng, Won Namgoong \\ Department of Electrical Engineering \\ University of Southern California \\ leifeng@usc.edu,namgoong@usc.edu
}

\begin{abstract}
An oversampled frequency channelized receiver for UWB radio in transmitted reference (TR) system is presented. Unlike previous work that assumes a white input noise, this paper includes the effects of the automatic gain controller (AGC) and the analog-to-digital converter (ADC) when large narrowband interference (NBI) is present. A detection method for the frequency channelized receiver when input noise is colored in TR UWB system is proposed. The proposed receiver significantly outperforms the full band receiver.
\end{abstract}

\section{INTRODUCTION}

Ultra-wideband (UWB) systems can coexist with narrowband radio systems by spreading the signal energy over a very wide bandwidth [1]. A major challenge in UWB communication is achieving accurate channel estimation. Transmitted reference (TR) modulation schemes has been proposed because of the ease of channel estimation in a dense multipath environment [2]. Optimal detection for UWB TR system was investigated in [3]. To achieve high performance, the UWB receiver needs to digitize the UWB signal at least at the signal Nyquist rate (usually several gigahertz) so that sophisticated digital detection schemes can be applied. Since designing a single $\mathrm{ADC}$ to operate at such sampling frequencies is difficult, parallel ADC architectures with each ADC operating at a fraction of the effective sampling frequency need to be employed.

To sample at a fraction of the effective sampling frequency, a frequency channelized receiver based on hybrid filter banks (HFB) (i.e., continuous-time analysis filters and discrete-time synthesis filters) has been proposed in [4]. Among the advantages of the frequency channelized receiver compared to the more conventional time channelized (i.e., time-interleaved ADC) receivers are the ease of designing the sample/hold circuitries, greater robustness to jitter/phase noise, and reduced ADC dynamic range requirements.

An oversampled frequency channelized UWB receiver was proposed in [5]. The analysis filters are designed to satisfy the power complementary condition so that the perfect reconstruction (PR) synthesis filters are the time-reversed complex conjugates of the analysis filters [6]. Since the matched filter is also the time-reversed complex conjugate of the propagation channel, the matched filter and the synthesis filter can be combined and the estimate of the optimal pulse response for detection can be obtained by simply averaging the combined responses of the propagation channel and the analysis filters.

While the channelized receiver described in [5] assumes a white input noise, NBI is a major challenge in UWB systems [7]. This paper extends the techniques in [5] to handle the effects of NBI (or colored noise) in TR UWB systems. The effect of the AGC and the ADC are also considered. By appropriately combining subband correlation values, the effect is to whiten and matched filter the received signal. As described in [4], an important advantage of the channelized receiver is that the effect of NBI can be isolated. Consequently, the ADC dynamic

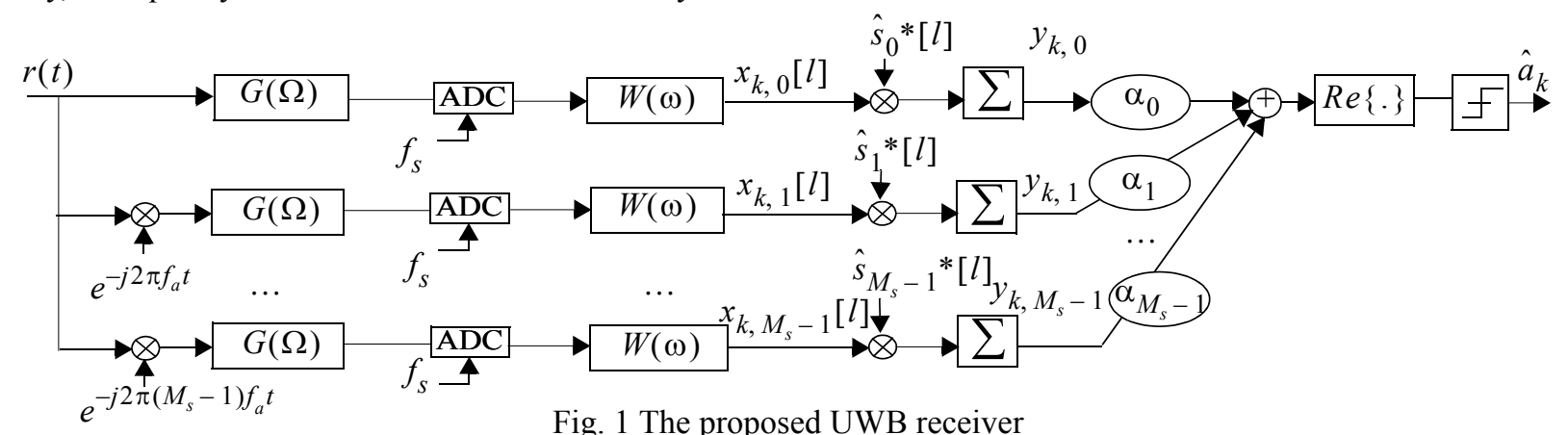

Fig. 1 The proposed UWB receiver 
range requirements is significantly relaxed compared to a full band receiver.

This paper is organized as follows. Section 2 introduces the receiver structure. In Section 3, AGC, ADC and NBI effects are considered. Simulation results are provided in Section 4 and conclusions are drawn in Section 5.

\section{RECEIVER STRUCTURE}

The UWB radio signal is an equally spaced pulse train with symbol period $T$. Sufficient guard time is introduced to prevent interference between symbols. The received UWB signal $r(t)$ is

$$
r(t)=\sum_{k} a_{k} s(t-k T)+v(t)
$$

where $a_{k}$ is the $k$ th transmitted antipodal symbol, $s(t)$ is the received signal pulse, and $v(t)$ is the additive white gaussian noise (AWGN).

A $M_{S}$ subband frequency channelized receiver is shown in Fig. 1. The received signal $r(t)$ is passed through a set of equally spaced mixers $e^{-j 2 \pi m f_{a} t}$, $m \in\left\{0,1, \ldots, M_{s}-1\right\}$. In each subband, the signal is filtered by a continuous-time analysis lowpass filter $G(\Omega)$, sampled and digitized by the ADC at a rate of $1 / T_{S}$, filtered by the discrete-time subband filter $W(\omega)$, and correlated with the conjugate of $\hat{s}_{m}[l]$, which is the estimate of the subband pulse response $s_{m}[l]$. The subband correlation values $y_{k, m}$ are weighted by $\alpha_{m}$ and summed to form $y_{k}$, which is the input to the slicer. If the received input noise is white, optimal detection is achieved by choosing $\alpha_{m}=1$ for $m \in\left\{1, \ldots, M_{s}-1\right\}$ and $\alpha_{0}=1 / 2$, since the received signal $r(t)$ is real. The effective sampling frequency of the HFB is $1 / T_{e}=\left(2 M_{s}-1\right) f_{a}$ and is related to the ADC sampling frequency $1 / T_{s}$ by $\gamma\left(\right.$ i.e., $T_{e}=$ $\left.T_{s} / \gamma\right)$ and to the frame frequency $1 / T$ by $N_{e}$ (i.e., $T_{e}=T / N_{e}$ ).

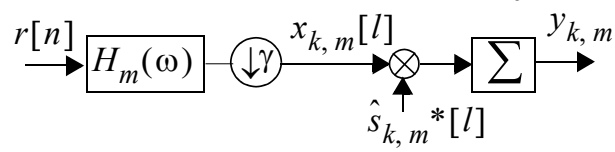

Fig. 2 The subband discrete model

A discrete equivalent model of the $m$ th subband is shown in Fig. 2. The received discrete UWB signal is $r[n]=r\left(n T_{e}\right)$. The discrete analysis filter $H_{m}(\omega)$ represents the combination of mixer $e^{-j 2 \pi m f_{a} t}$, continuous-time lowpass filter $G(\Omega)$, and discrete-time subband filter $W(\omega)$. The ADC is modeled as a downsampler with rate $\gamma$. To form a complete representation of the received signal, $M=2 M_{s}-1$ subbands between 0 and $2 \pi$ are needed because the discrete spectrum covered by the $M_{s}$ subbands is mainly between 0 and $\pi$. Since the received signal is assumed to be real, the remaining spectrum is covered by conjugating signals in the 1 st to $\left(M_{s}-1\right)$ the subbands. These subbands are virtual subbands as they are not actually implemented but are used to simplify the analysis. For the above reason, we assume $m \in\{0, \ldots, M-1\}$.

The optimal detection conditions are derived by cascading a PR filter bank and the matched filter. The channelized signals are first reconstructed by passing through PR synthesis filters. The reconstructed $r[n]$ is then filtered by a matched filter for optimal detection. When the synthesis filter is chosen to be the complex conjugate of $H_{m}(\omega)$ (i.e., $\left.H_{m}{ }^{*}(\omega)\right)$ to simplify the estimation process, optimal detection is achieved if

$$
\begin{gathered}
\sum_{m=0}^{M-1} H_{m}(\omega-2 \pi \nu / \gamma) H_{m}{ }^{*}(\omega)=0 \\
\quad M-1 \\
\sum_{m=0}\left|H_{m}(\omega)\right|^{2}=\gamma
\end{gathered}
$$

for $v=1, \ldots, \gamma-1$ and all $\omega$. The first condition given in (2) can be satisfied by operating the HFB above the critically sampling rate. The second condition given in (3) is satisfied by ensuring that $\left\{H_{m}(\omega)\right\}$ satisfies the power complementary condition. This is achieved by designing the digital filter $W(\omega)$ to satisfy the power complementary condition and ensuring (by sufficiently oversampling) that the overall amplitude response of $G(\Omega)$ and $W(\omega)$ is approximately $|W(\omega)|$ despite uncertainties in the analog circuitries from process and temperature variations. By designing $\left\{H_{m}(\omega)\right\}$ to satisfy conditions (2) and (3), the matched filter and the synthesis filter can be combined and the optimal detection is achieved by correlating in each subband with subband template signal $\hat{s}_{m}{ }^{*}[l]$, which can be estimated by averaging the received subband signal.

\section{AGC, ADC and NBI}

\subsection{Detection with $A G C$}

In the previous sections, the effect of the ADC and the AGC were not considered. To fully utilize the dynamic range of the ADCs, an AGC is applied in each subband channel so that the percentage of the signal overflowing (or clipping) is kept within a certain percentage. This section describes how to account for the effect of the AGC when using the channelized receiver for detecting in UWB TR systems.

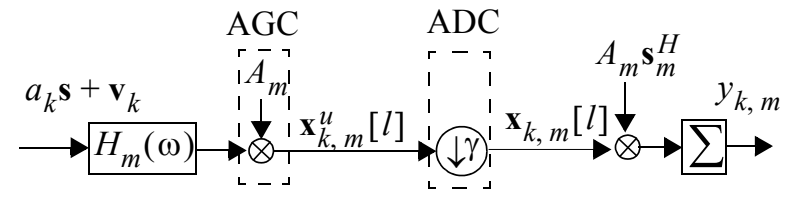

Fig. 3 AGC in the $m$ th subband 
The $k$ th received frame is in a vector form $a_{k} \mathbf{s}+\mathbf{v}_{k}$, where $\mathbf{s}$ is the signal component and $\mathbf{v}_{k}$ is noise. The $m$ th subband with an AGC is shown in Fig. 3. The AGC gain $A_{m}$ is set so that the power of $\mathbf{x}_{k, m}^{u}$, which is the signal and noise at the input of the ADC, is kept constant. The frame index $k$ is dropped in $A_{m}$ since the power of $\mathbf{x}_{k, m}^{u}$ is assumed constant within a transmission block. The downsampled subband signal is

$$
\mathbf{x}_{k, m}=a_{k} A_{m} \mathbf{s}_{m}+A_{m} \mathbf{v}_{k, m}
$$

where $\mathbf{s}_{m}$ is the subband template signal and $\mathbf{v}_{k, m}$ is the subband noise in the $k$ th frame. Assuming ideal subband pulse response estimation, the subband correlation signal is $A_{m} \mathbf{s}_{m}^{H}$. The subband correlation value is

$$
y_{k, m}=a_{k} A_{m}^{2} \mathbf{s}_{m}^{H} \mathbf{s}_{m}+A_{m}^{2} \mathbf{v}_{k, m}^{H} \mathbf{s}_{m}
$$

For optimal detection, the correlation value in each subband channel needs to be weighted to remove the effect of the AGC before summing, i.e.,

$$
y_{k}=\sum_{m=0}^{M-1} \alpha_{m} y_{k, m}
$$

where

$$
\alpha_{m}=K / A_{m}^{2}
$$

and $K$ is a constant.

In TR systems, $\alpha_{m}$ can be estimated from the subband noise $A_{m} \mathbf{v}_{k, m}$. Since $\mathbf{v}_{k, m}$ is obtained by passing a white noise $\mathbf{v}_{k}$ through a set of filters $H_{m}(\omega)$, which is the modulated version of a prototype filter, the noise power of $\mathbf{v}_{k, m}$ has the same value of $\sigma_{s}^{2}$ in all subbands. Hence, $\alpha_{m}$ can be expressed as

$$
\alpha_{m}=\frac{1}{E\left\{\left|A_{m} \mathbf{v}_{k, m}\right|^{2}\right\}}=\frac{1 / \sigma_{s}^{2}}{A_{m}^{2}}
$$

$E\left\{\left|A_{m} \mathbf{v}_{k, m}\right|^{2}\right\}$ can be obtained by estimating the variance of $\mathbf{x}_{k, m}$ in (4) based on the first $N_{r}$ training symbols. Using the estimate of the subband pulse response signal

$$
\hat{\mathbf{s}}_{m}=\frac{1}{N_{r}}\left(\sum_{k=1}^{N_{r}} \mathbf{x}_{k, m}\right)
$$

the estimate of the variance of $\mathbf{x}_{k, m}$ is

$$
\begin{aligned}
& \hat{\sigma}_{m}^{2}=\frac{1}{N_{r}-1} \sum_{k=1}^{N_{r}}\left(\mathbf{x}_{k, m}-\hat{\mathbf{s}}_{m}\right)^{H}\left(\mathbf{x}_{k, m}-\hat{\mathbf{s}}_{m}\right) \\
& =\frac{1}{N_{r}-1}\left[\sum_{k=1}^{N_{r}} \mathbf{x}_{k, m}^{H} \mathbf{x}_{k, m}-\frac{1}{N_{r}}\left(\sum_{k=1}^{N_{r}} \mathbf{x}_{k, m}\right)^{2}\right]
\end{aligned}
$$

\subsection{Detection with colored noise and NBI}

When NBI or colored noise is present, optimal detection is achieved by first whitening the noise then matched fil- tering the resulting signal. In channelized receivers, the whitening operation can be performed by appropriately weighting the subbands. The colored noise can be approximately modeled as a white noise weighted by different coefficients $\left\{B_{m}\right\}$ in different subbands as in Fig. 4. The input to the $m$ th subband channel is then viewed as being $\mathbf{s} / B_{m}$ instead of $\mathbf{s}$. Since the input noise in this model is white, optimum detection is achieved as described in the previous subsection.

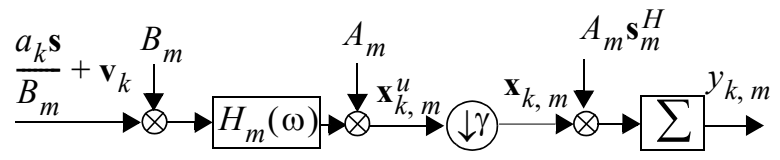

Fig. 4 Colored noise and NBI model

The downsampled subband signal is

$$
\mathbf{x}_{k, m}=a_{k} A_{m} \mathbf{s}_{m}+A_{m} B_{m} \mathbf{v}_{k, m}
$$

Correlating with $A_{m} \mathbf{s}_{m}^{H}$ (or its estimate in practice), the resulting subband correlation value is

$$
y_{k, m}=a_{k} A_{m}^{2} \mathbf{s}_{m}^{H} \mathbf{s}_{m}+A_{m}^{2} B_{m}^{2}\left(\mathbf{s}_{m}^{H} / B_{m}\right) \mathbf{v}_{k, m}
$$

Comparing (13) with (5), the only difference is that $\mathbf{s}_{m}$ and $A_{m}$ is replaced by $\mathbf{s}_{m} / B_{m}$ and $A_{m} B_{m}$, respectively. Hence, from (7), the weighting in each subband after correlation is

$$
\alpha_{m}=\frac{K}{A_{m}^{2} B_{m}^{2}}
$$

Recalling (8) and replacing $A_{m}$ with $A_{m} B_{m}$, the estimate of $\alpha_{m}$ in colored noise and NBI can also be obtained by estimating subband noise variance with (10) and (11).

\section{SIMULATION RESULTS}

The transmitted monocycle is the second derivative of a gaussian pulse, i.e.,

$$
p(t)=\left(1-4 \pi t^{2}\right) \exp \left(-2 \pi t^{2}\right)
$$

The received noise free signal pulse is a superposition of monocycles with different delays and amplitudes. The multipath model used is the CM1 channel model recommended by the IEEE P802.15-02/368r5-SG3a. The effective sampling frequency is $1 / T_{e}=3.5 \mathrm{GHz}$.

Assuming $M_{s}=4$ and $\gamma=5$, the ADC sampling rate is $7 / 5$ times the critically sampling rate. The low pass filter $G(\Omega)$ in the analysis filters is an eighth order Butterworth filter, and its $3 d B$ frequency is at $1 /\left(12 T_{e}\right) . W(\omega)$ is obtained from [8]. The received symbol energy is $E_{b}=\mathbf{s}^{H} \mathbf{s}$ and the white channel noise power density is $N_{0}$.

Fig. 5 shows the bit error rate (BER) for a TR system when $s_{m}{ }^{*}[l]$ is unknown. The first one or four training symbols in a block of 100 symbols is used to estimate the time varying $s_{m}{ }^{*}[l]$. The estimation is then used to detect the remaining symbols in the block. The performance of an ideal matched filter receiver with perfect knowledge of the 
received signal pulse is shown as the lower bound. A full band receiver, which samples the UWB signal with a single $\mathrm{ADC}$ at the effective sampling rate and estimates the signal pulse by averaging the training symbols, is used for comparison. As the number of training symbols increases, the performance of both receivers approach that of the ideal receiver.

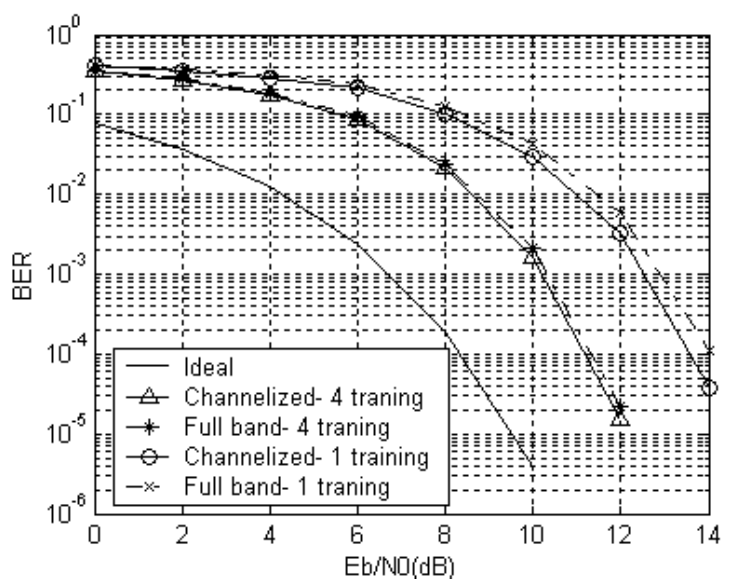

Fig. 5 Ideal BER performance

To account for the effects of the AGCs, subband correlation noise powers are estimated as in (11) and used to weigh the subband correlation values. Using four training symbols, the BER performances with 1-bit and 3-bit ADCs are shown in Fig. 6 . The receiver slightly outperforms the full band receiver especially for low ADC resolution.

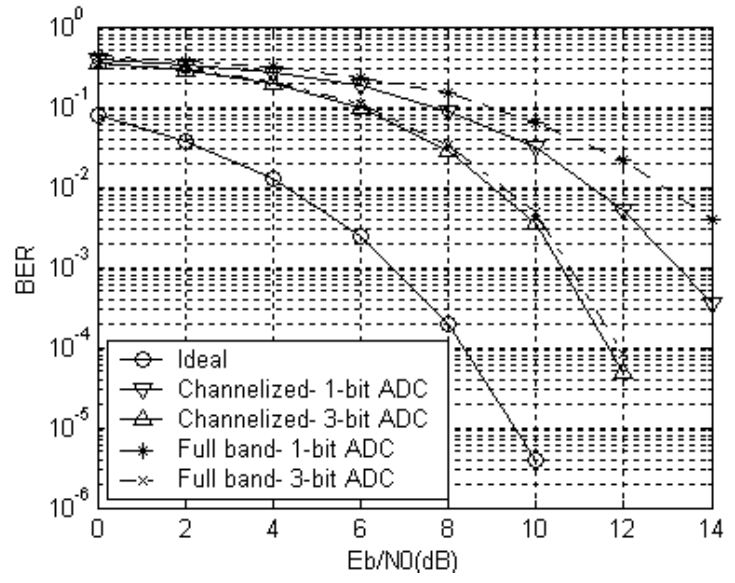

Fig. 6 AGC and ADC

NBI is measured in terms of the signal-to-interference ratio (SIR), which is defined as the ratio between the received symbol energy $E_{b}$ and the interference power. The NBI is generated by filtering a template white noise with a sharp bandpass filter whose bandwidth is 0.05 of the signal bandwidth. Four training symbols are employed in the following NBI simulations. In Fig. 7, SIR $=-5 d B$, and the NBI center frequency is $0.57 f_{e} / 2$, which corresponds to the frequency with the strongest signal power. Regardless of $E_{b} / N_{0}$ values, the full band receiver ceases to function, but the channelized receiver performs well even when low resolution ADCs are employed.

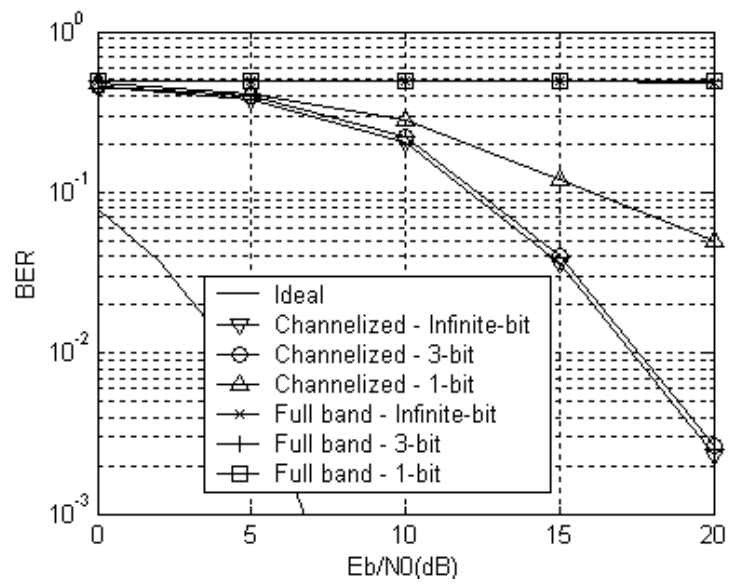

Fig. 7 NBI with $S I R=-5 d B$

Fig. 8 shows BER at different SIRs given $E_{b} / N_{0}=15 d B$. The NBI center frequency is $0.57 f_{e} / 2$. For the same BER performance, the channelized receiver can tolerate much stronger NBI than the full band receiver. The proposed channelized receiver, therefore, achieves greater robustness to NBI.

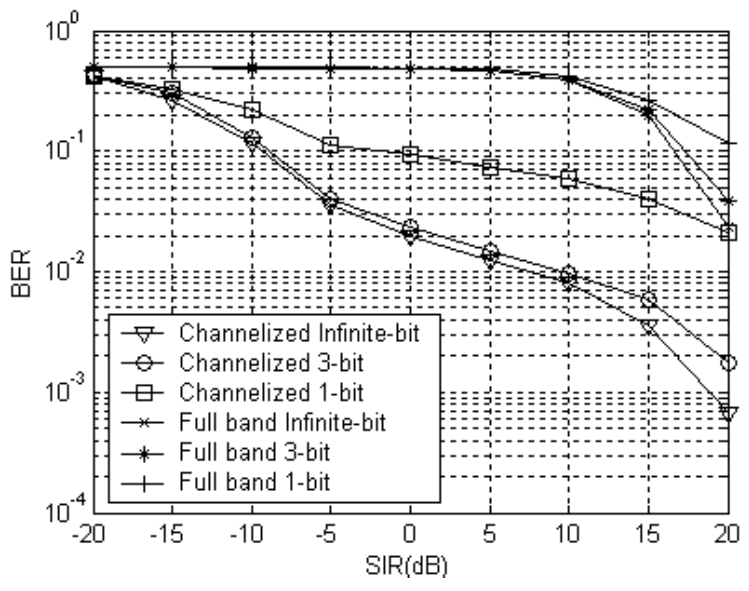

Fig. 8 BER v.s. SIR

\section{CONCLUSION}

An oversampled channelized receiver for UWB radio TR systems is investigated in the presence AGC, ADC, and NBI. If strong narrowband interferer is present, the proposed receiver significantly outperforms the full band receiver 
because of its ability to isolate the effect of the NBI. Our results suggests a low resolution ADC (i.e., 3-bits) is adequate for the proposed receiver even in the presence of large NBI.

\section{ACKNOWLEDGEMENT}

This work was supported in part by the Army Research Office under Contract DAAD19-01-1-0477 and the National Science Foundation under Contract ECS-0134629.

\section{REFERENCES}

[1] M. Z. Win and R. A. Scholtz, "Impulse Radio: How It Works," IEEE Comm. Letters, vol. 2, NO. 2, pp. 36-38, Feb. 1998

[2] R. Hoctor and H. Tomlison, "An Overview of DelayHopped, Transmitted-Reference RF Communications," GE Research and Development Center, Tech. Rep. 2001CRD198, 2002
[3] S. Franz and U. Mitra, "On Optimal Data Detection for UWB Transmitted Reference Systems," IEEE GLOBECOM 2003, pp744-748, 2003

[4] W. Namgoong, "A Channelized Digital Ultra-Wideband Receiver,” IEEE Trans. Wireless Comm., vol. 2, pp. 502510, May 2003

[5] L. Feng and W. Namgoong, "An Oversampled Channelized UWB Receiver,” UWBST 2004.

[6] R. E. Crochire and L. R. Rabiner, "Multirate Digital Signal Processing," Prentice Hall, 1983

[7] R.A. Scholtz etc. "UWB Radio Deployment Challenges," PIMRC 2000, Sept. 2000

[8] M. Harteneck, S. Weiss, and R.W. Stewart, "Design of Near Perfect Reconstruction Oversampled Filter Banks for Subband Adaptive Filters," IEEE Trans. on Circuits and Systems Part II: Analog and Digital Signal Processing, Vol.46, No.8, pp.1081-1086, Aug. 1999 